\title{
A Problem on Determination of Hydrogen in Steel Using Graphite Crucible*
}

\section{By Yoshihide ENDO, ,* Yasuharu MATSUMURA** and Noritaka SAKAO**}

\begin{abstract}
Synopsis
For the determination of hydrogen in steel, recently the high-frequencyfusion or impulse-furnace-fusion method using the graphite crucible is used on account of their rapidity. Comparing with the conventional heat-extraction method using no graphite crucible, the above new methods are inclined to give higher hydrogen contents. In the quenched samples, fine cracks and blow-holes are often contained where water is included during the storage and preparation of samples. As the result of the reaction between the included water and the graphite crucible (water gas reaction), the evolved hydrogen is accumulated to the hydrogen result,

For elimination of the included water, the authors invented the pre-heat device. The included water in samples can be eliminated by heating at $300^{\circ}$ to $350^{\circ} \mathrm{C}$ and at the same time, the diffused hydrogen is collected to correct the final result.

Using the improved method the determination of hydrogen is carried out exactly, even if the sample contains some blow-holes. This new method requires only $8 \mathrm{~min}$ for one determination and gives the good accuracy (standard deviation $=0.1 \mathrm{ppm} \mathrm{H}_{2}$ at $2.5 \mathrm{ppm}$ ).
\end{abstract}

\section{Introduction}

There are many methods ${ }^{1)}$ for the determination of hydrogen content in steel, but recently the highfrequency-fusion method and the impulse-furnacefusion method ${ }^{2,3)}$ using a graphite crucible are widely used for their rapidity of determination. However, in case of the quenched samples ${ }^{4)}$ the values determined by these new methods were mostly higher than the ones by other methods with no graphite crucible, e.g., the heat-extraction method, etc. However, there was no difference between the determined values in case of other standard samples with no flaws.

Therefore, the difference between the values by the method with and without, the graphite crucible was considered due to the water penetrated in the fine cracks and blow-holes of the sample at the time of cooling-down after sampling and during preparation with coolant such as dryice and alcohol. The extra hydrogen would be generated by the reaction between the graphite crucible and the included water (water gas reaction).

A method is reported in this paper for the elimination of the hydrogen generated by this water gas reaction.

\section{Instrumentation}

The following two instruments were used. A LECO RH-1E was used as the new fusion method with graphite crucible, and a Naruse Kagakukiki Prof. Gotoh's GHN-1 ${ }^{5)}$ for the heat-extraction method with no graphite crucible. The former is called for short the impulse-furnace-fusion method, the latter is called the heat-extraction method.

\section{Impulse-furnace-fusion Method}

The sample is fused in the graphite crucible and the released hydrogen is determined by the gaschromatography. The procedure of this method is as follows:

The sample in the graphite crucible is fused with the impulse furnace at temperature of about $2200^{\circ} \mathrm{C}$ in a constant flow of argon. The released gases are passed through iodine pentoxide so that carbon monoxide is oxidized to dioxide, which is absorbed by ascarite. Nitrogen and hydrogen are separated by a molecular sieve column. The hydrogen is introduced to a thermal conductivity detector and measured. The amplified and integrated signal is displayed on a digital voltmeter, and this value is converted to the content of hydrogen by the use of a calibration curve.

Operating conditions

Ar gas flow rate

Reference gas:

$50 \mathrm{ml} / \mathrm{min}$

Measuring gas:

$180 \mathrm{ml} / \mathrm{min}$

(1) Outgassing process

Outgassing temperature: $\quad 2700^{\circ} \mathrm{C}$

Outgassing time:

$70 \mathrm{sec}$

(2) Analysis process

Sample weight:

Combustion accelerator:

$3 \sim 4 \mathrm{~g}$

Analysis temperature:

Sn $(2 \mathrm{~g})$

$2200^{\circ} \mathrm{C}$

$90 \mathrm{sec}$

Fusion time:

$190 \mathrm{sec}$

\section{Heat-extraction Method}

In this method, the sample is heated under inert gas flow, and then the hydrogen is separated from the released gases through a heated palladium tube and determined. The procedure is as follows.

The sample is charged in a quartz tube of about $1000^{\circ} \mathrm{C}$ under constant flow of argon carrier gas. The extracted hydrogen and other gases are passed through the palladium tube heated at about $800^{\circ} \mathrm{C}$ to separate the hydrogen. The pressure and volume of the hydrogen is measured, and then converted to the content in steels.

\section{Operating conditions}

Ar gas flow rate:

Sample weight:

$80 \mathrm{ml} / \mathrm{min}$ $3 \sim 7 \mathrm{~g}$

* Originally published in Tetsu-to-Hagané, 63 (1977), 2391, in Japanese. English version received August 25, 1977.

** Mizushima Works, Kawasaki Steel Corp., Mizushima Kawasaki-dori, Kurashiki 712. 
Temperature in quartz tube:

$1000^{\circ} \mathrm{C}$

Temperature of sponge titanium column:

Temperature of palladium tube:

$500^{\circ} \mathrm{C}$

$800^{\circ} \mathrm{C}$

\section{Experimental and Discussion}

\section{Determination by the Impulse-furnace-fusion Method and Its Problem}

Figure 1 gives the hydrogen contents in the quenched sample and the standard sample determined by the impulse-furnace-fusion method and the heatextraction method. The samples quenched in the mold had few cracks and blow-holes. It is shown in Fig. 1 that the values of the standard samples had no difference between two methods, but in case of the mold samples, the values by the impulse-furnacefusion method were higher than ones by the heatextraction method, $8 \mathrm{ppm}$ higher in extreme case.

\section{Discussion about the Cause of the Difference}

As mentioned above, the results of the samples quenched in the mold had a large difference between two methods. In order to clarify the cause of the difference, the following experiments were made.

(1) Determination of the Hydrogen Remained after Heat-extraction

It was expected that the lower heating temperature of the extraction method caused the lower value. Thus the sample, already determined by the heat-

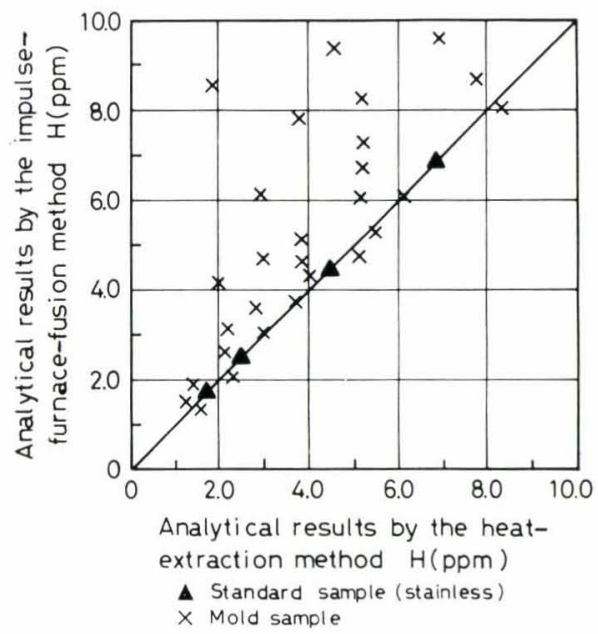

Fig. 1. Comparison of analytical results by the impules-furnace-fusion and the heat-extraction method extraction method, was again determined by the impulse-furnace-fusion method. However, the remained hydrogen was not detected. So, it was proved that the hydrogen was completely extracted by the heatextraction method.

(2) Determination of the Primary and the Secondary Hydrogen

The primary hydrogen was determined with microOrsat on the sample kept in a mercury bell ${ }^{6}$ during five days at room temperature. After the primary hydrogen was determined, the secondary hydrogen of the same sample was determined by the impulsefurnace-fusion method. The determined secondary hydrogen was added to the primary hydrogen, and then the primary and secondary hydrogen content was compared with the result by the heat-extraction method. Table 1 gives the result, and the former agreed with the latter.

As the result of items (1) and (2), it seemed that the results by the heat-extraction method were accurate, so that the difference of the results between two methods would be due to the impulse-furnace-fusion method.

(3) Influence of the Included Water

The water in the cracks and blow-holes of the quenched sample was considered to be the cause of the higher result by the impulse-furnace-fusion method. Stainless steel samples had no difference between two methods, probably because they could be made in the shape of plate and of stick with no crack and blow-hole. However, in case of the quenched sample, the cracks and blow-holes were found on the surface of many samples. The penetrated water in the cracks and blow-holes would react with the graphite crucible, when the impulse-furnacefusion method was used. The hydrogen generated by the water gas reaction will be the cause of the positive error. The crack and the blow-hole are so fine, unable to be found out without a microscope.

Thus, in order to investigate the influence of the water in the cracks and blow-holes, the sample with the blow-holes was used to extract gases by the heatextraction method. Then this sample was dipped in water, and washed with ether. The hydrogen was again determined by the impulse-furnace-fusion method. Table 2 gives the result. The hydrogen content was increased clearly by the water in the cracks and blow-holes.

Table 1. Comparison of the result by the successive method with the one by the heat-extraction method

\begin{tabular}{|c|c|c|c|c|c|}
\hline \multirow[b]{2}{*}{ Sample } & \multicolumn{3}{|c|}{ Successive method $\mathrm{H}(\mathrm{ppm})$} & \multirow{2}{*}{$\begin{array}{c}\text { Heat-extraction } \\
\text { method (2) } \\
\text { H (ppm) }\end{array}$} & \multirow{2}{*}{$\begin{array}{l}\text { Difference } \\
(1)-(2) \\
\text { H (ppm) }\end{array}$} \\
\hline & $\begin{array}{c}\text { Primary } \\
\text { hydrogen* }\end{array}$ & $\begin{array}{c}\text { Secondary } \\
\text { hydrogen** }\end{array}$ & Total (1) & & \\
\hline Plain carbon steel & 2.63 & 0.65 & 3.28 & 3.15 & +0.13 \\
\hline Plain carbon steel & 1.17 & 0.33 & 1.50 & 1.54 & -0.04 \\
\hline Low alloy steel & 4.98 & 0.64 & 5.62 & 5.85 & -0.23 \\
\hline
\end{tabular}

* Primary hydrogen: Diffusive hydrogen at room temperature

** Secondary hydrogen: Extracted hydrogen at high temperature after diffusive hydrogen determination 
Table 2. Effect of included water in the de-hydrogen sample on hydrogen determination

\begin{tabular}{c|cc}
\hline States of the sample & $\begin{array}{c}\text { Analytical results by the } \\
\text { impulse-furnace fusion method } \\
\text { H (ppm) }\end{array}$ \\
\hline $\begin{array}{c}\text { Sample containing cracks and } \\
\text { blow-holes abundantly }\end{array}$ & 3.5 & 6.5 \\
$\begin{array}{c}\text { Sample containing fine cracks } \\
\text { and blow-holes }\end{array}$ & 1.9 & 0.6 \\
$\begin{array}{c}\text { Sample containing no crack } \\
\text { and blow-hole }\end{array}$ & 0.2 & 0.1 \\
\hline
\end{tabular}

\section{Discussion about a Method for Elimination of the} Water in the Cracks and Blow-holes

As mentioned above, the quenched sample would contain the water in the cracks and blow-holes, and if so, the water should affect the determined value of the hydrogen. To prevent the influence of the water, the following three methods are proposed.

1) Dry with hot wind by a blower ${ }^{\text {) }}$

2) Wash with acetone

3) Heat in the impulse furnace

The first method has the possibility to release the hydrogen in the steel sample. The second method is effective to separate the water adhering on the sample surface, but uneffective to the water in the deep cracks and blow-holes. The third method is impossible to remove the separated water completely out of measuring system, the remained water will affect the result. Therefore a pre-heat device was made as the fourth method. By using the pre-heat device, the water was removed out of the measuring system. The released hydrogen during the pre-heat was collected and determined.

\section{Determination of the Hydrogen with the Pre-heat Device}

\section{Improvement of the Impulse-furnace-fusion Method}

As shown in Fig. 2, the pre-heat device was attached to LECO RH-1E. Figure 3 shows the preheat device consisting of a sample heat part and a reagent column. The procedure is as follows:

The sample is kept by (2) in Fig. 3, argon carrier gas purges the quartz tube (1) and the reagent column. At the same time, the graphite crucible is outgassed with the impulse furnace. Then, the sample is moved to (3), and heated at temperature of about $350^{\circ} \mathrm{C}$. The vaporized water is absorbed with the reagent column (4), and the simultaneously released hydrogen is introduced to the detector, the signal of the hydrogen is integrated. Then, the sample is moved into the graphite crucible (5), fused with the impulse furnace. The signal of the released hydrogen is integrated. The former and latter integrated value is converted to the content by the calibration curve.

The optimum pre-heating temperature was $300^{\circ}$ to $350^{\circ} \mathrm{C}$. When the temperature was below $300^{\circ} \mathrm{C}$, the time for the water separation was too long. In case of higher temperature than $350^{\circ} \mathrm{C}$, the decomposition of the water affected the result. The integration time was $240 \mathrm{sec}$.

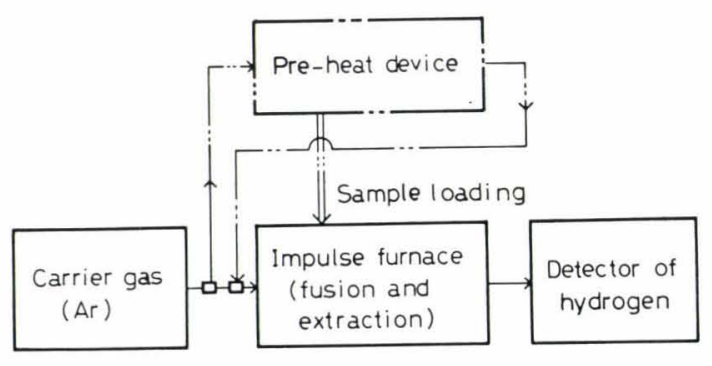

Fig. 2. Block diagram of the improved apparatus

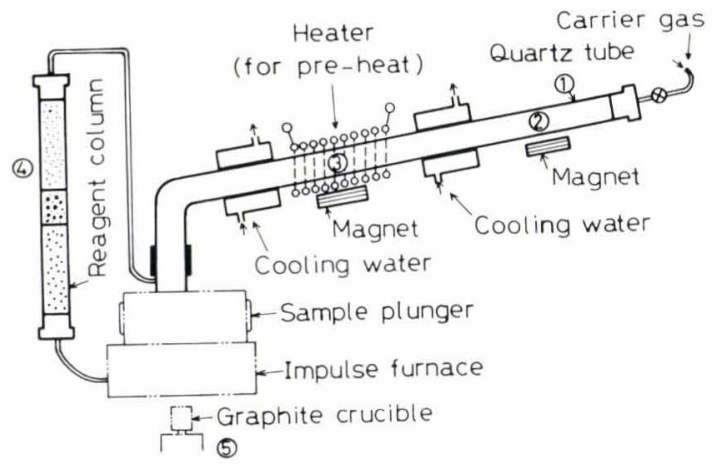

Fig. 3. Detail of the pre-heat device

\section{Result of Determination}

Figure 4 gives the result of the determination of the hydrogen content in the samples quenched in the mold and the standard samples by the improved impulse-furnace-fusion method. The results by the improved method agreed with the values by the heatextraction method also even in case of the quenched samples. Thus, it was recognized that this method was not affected by the water in the cracks and blow-holes.

Further, the ratio of the released hydrogen by preheating to the total hydrogen was studied. The used samples were carbon steel. The ratio was $70 \%$ by pre-heating at $350^{\circ} \mathrm{C}$ for $90 \mathrm{sec}$ and $80 \%$ at $350{ }^{\circ} \mathrm{C}$ for $150 \mathrm{sec}$.

The reproducibility of the results of the mold sample by the improved method was $0.1 \mathrm{ppm}$ as standard deviation at hydrogen content of $2.5 \mathrm{ppm}$. Compared with the reproducibility by the heat-extraction method of its standard deviation of $0.2 \mathrm{ppm}$, the improved method has the better accuracy. The improved method requires $8 \mathrm{~min}$ for one determination.

\section{Segregation in the Sample}

The better reproducibility of the improved method enabled a study of the segregation in the mold sample. Figure 5 shows an example. The sample was devided into sixteen parts and the hydrogen of each part was determined. There was little difference between each result and also between various types of steel. The standard deviation of the segregation in the sample was between $0.07 \mathrm{ppm}$ and $0.11 \mathrm{ppm}$ for hydrogen content between $1.5 \mathrm{ppm}$ and $6 \mathrm{ppm}$, respectively.

\section{Determination of the Sample with Many Cracks and Blow-holes}

Some quenched samples have many cracks and 


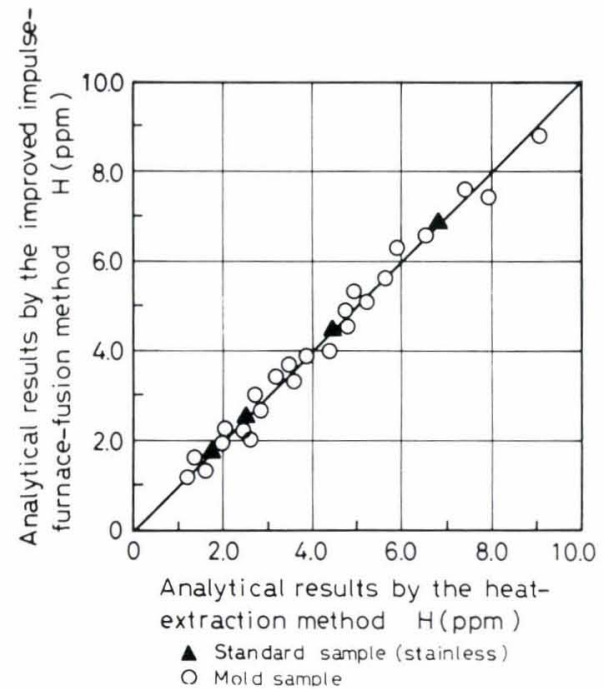

Fig. 4. Comparison of analytical results by the improved impulse-furnace-fusion and the heat-extraction method

blow-holes. These samples have possibility to contain water and the results by the impulse-furnacefusion method are extremely high.

The heat-extraction method is not affected by the water in the cracks and blow-holes, when the sample contains a little water. However, in case of the sample containing much water, the measuring pressure sometimes keeps on increasing gradually. This phenomenon is probably caused by the decomposition of the water adhered on the palladium tube and makes the end point of extraction indistinct. Figure 6 shows the result of the samples with many cracks and blow-holes. The results by the impulse-furnacefusion method were between $20 \mathrm{ppm}$ and $30 \mathrm{ppm}$, while the results by the heat-extraction method were between $10 \mathrm{ppm}$ and $15 \mathrm{ppm}$. However, the results by the improved impulse-furnace-fusion method were between $5.5 \mathrm{ppm}$ and $6.3 \mathrm{ppm}$. These values agreed with the result of the sample with few cracks and blow-holes which was sampled simultaneously. The standard deviation was $0.25 \mathrm{ppm}$ for this experimentation.

\section{Conclusion}

(1) The hydrogen content in steel was determined by the impulse-furnace-fusion method and the heatextraction method. In case of the sample quenched in the mold, the result by the former method tends to be higher than the result by the latter.

(2) The graphite crucible is the cause of it. Almost all the "mold" samples have the cracks and blow-holes. Water penetrates into them and the water reacts with the graphite and the generated hydrogen is added to the hydrogen in steel. The graphite crucible is necessary for the impulse-furnacefusion method, so this method is influenced by the
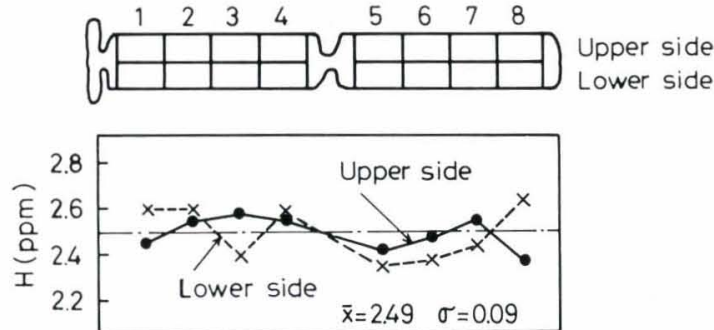

Fig. 5. Example of the segregation in mold sample

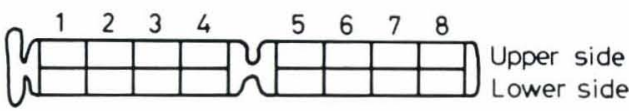

States of blow-holes and cracks
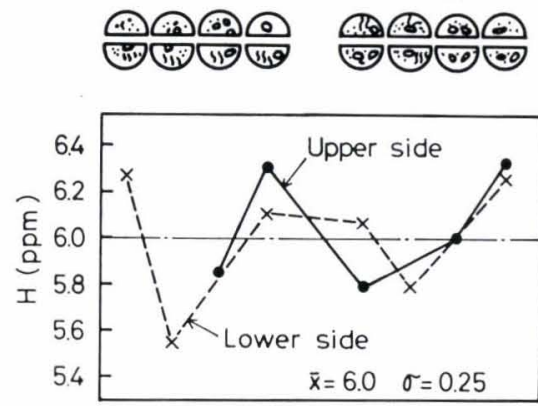

Fig. 6. Example of the segregation in mold sample containing cracks and blow-holes abundantly

water in the cracks and blow-holes.

(3) To prevent the influence by the water, the pre-heat device was made. By the use of the improved impulse-furnace-fusion method, the water can not affect the result, even when the graphite crucible is used. The determination method for the hydrogen content in steel was established.

(4) The improved method required about $8 \mathrm{~min}$ for one determination. The standard deviation was $0.1 \mathrm{ppm}$, when the hydrogen content of the used sample was $2.5 \mathrm{ppm}$.

\section{REFERENCES}

1) JIS Z-2614, (1973).

2) Y. Endo, Y. Matsumura and N. Sakao: Tetsu-to-Hagané, 59 (1973), S623.

3) Y. Endo and N. Sakao: Analytical Instruments, 11 (1973), 35.

4) The 19th Committee on Steelmaking, The Japan Society for the Promotion of Science: Tekkoh Kagaku Zensho, VIII, Nikkan Kogyo Shinbunsha, Tokyo, (1963), 77.

5) H. Gotoh, S. Ikeda and N. Hosoya: Report of the 19th Committee of the Japan Society for the Promotion of Science, Report No. 7224, (1963).

6) S. Oda: Report of the 19th Committee of Japan Society for the Promotion of Science, Report No. 7226, (1963).

7) I. Ogawara and M. Ishikawa: Report of the 19th Committee of Japan Society for the Promotion of Science, Report No. 9548, (1973). 